\title{
Magnetic Particle Trapping in a Branched Blood Vessel in the Presence of Magnetic Field
}

\author{
Samia F. Salem ${ }^{1,2^{*}}$ and Valery V. Tuchin ${ }^{1,3,4,5}$ \\ ${ }^{1}$ Department of Optics and Biophotonics, Saratov State University, 83 Astrakhanskaya str., Saratov 410012, Russia \\ ${ }^{2}$ Department of Physics, Faculty of Science, Benha University, Egypt \\ ${ }^{3}$ Interdisciplinary Laboratory of Biophotonics, National Research Tomsk State University, 36 Lenin Avenue, \\ Tomsk 634050, Russia \\ ${ }^{4}$ Laboratory of Molecular Imaging, Bach Institute of Biochemistry, Research Center of Biotechnology of the Russian \\ Academy of Sciences, Moscow 119071, Russia \\ ${ }^{5}$ Institute of Precision Mechanics and Control of the Russian Academy of Sciences, Saratov 410028, Russia \\ * e-mail: samiafarouk66@yahoo.com
}

\begin{abstract}
This study presents a theoretical model by using the COMSOL Multiphysics ${ }^{\circledR}$ software to describe the behavior of magnetic nanoparticles through blood stream in a branched blood vessel under the influence of cylindrical permanent magnet that is located outside the vessel. The magnet is placed at one branched vessel to attract the magnetic particles towards targeted locations. The fluid (blood) is assumed being Newtonian; its flow is incompressible and laminar. Magnetic nanoparticles, such as superparamagnetic iron oxide $\left(\mathrm{Fe}_{3} \mathrm{O}_{4}\right)$ nanoparticles are used in this theoretical study. The mechanisms of magnetic nanoparticles travelling in the blood stream under influence of a localized static magnetic field are numerically studied. The equations of motion for particles in the flow are governed by a combination of magnetic equations for the permanent magnetic field and the Navier-Stokes equations for fluid. (C) 2020 Journal of Biomedical Photonics \& Engineering.
\end{abstract}

Keywords: magnetic nanoparticles; branching vessels; permanent magnet; magnetism; Newtonian fluid; blood vessel; computational modeling.

Paper \#3395 received 17 Nov 2020; revised manuscript received 11 Dec 2020; accepted for publication 12 Dec 2020; published online 31 Dec 2020. doi: 10.18287/JBPE20.06.040302.

\section{Introduction}

Nanotechnology has gained a great deal of attention for its potential use in medical research. In different biomedical applications, iron oxide nanoparticles have shown a promising effect. In particular, magnetite $\left(\mathrm{Fe}_{3} \mathrm{O}_{4}\right)$ nanoparticles with size ranging from 1 to $1000 \mathrm{~nm}$ are commonly used because of their biocompatibility, high magnetic susceptibility, chemical stability, innocuousness, high saturation magnetization $[1,2]$. They are mostly used in the biomedical field, e.g., greater surface-to-volume ratio, high magnetic characteristics, high activity, and new optical properties because of their superior nature over pure-sized particles. Among cells and biomolecules, nanoparticles have high applicability. The agglomeration, charge, chemical composition, crystalline structure, form, size and solubility of nanoparticles affect interactions with cells and biomolecules $[3,4,5]$. Nowadays magnetic nanoparticles are able to design integration for a broad variety of biomedical applications, such as analytical devices, bioimaging, biosensors, contrast agents (CAs), hyperthermia, photoablation therapy, applications for physical therapy, separation and delivery of targeted drugs (TDD) [1-8].

The work by Sindeeva et al. [9] aims to establish the processes that occur in the bloodstream following the systemic injection of $5 \mu \mathrm{m}$ polyelectrolyte capsules with magnetic nanoparticles in the membrane. It has been shown that the amount of circulating capsules decreases several times in 1 min after injection, and that less than $1 \%$ of the injected dose is reported in the blood after $15 \mathrm{~min}$. At this time, much of the capsules build up in the lungs, liver, and the kidneys. However, magnetic field 
activity in the region-of-interest may increase the accumulation of capsules slightly. After intravenous injection of microcapsules, they examined real-time blood flow changes in vital organs in vivo using a laser speckle contrast imaging system. They have shown the organism can respond to the presence of drug carriers in the blood and their accumulation in the vessels of vital organs.

Zhang et al. [10] provided a numerical simulation of the magnetic targeting of a drug using ANSYS 19.1 software that explores an external magnetic field to load drugs with magnetic nanoparticles for a patient-specific atherosclerotic plaque. The magnetic field generated from the wire with current; the wire form and the magnetic field are the key parameters to deliver the drug to the target (atherosclerotic plaque). Blood inlet velocity is the short-term velocity $u(t)$, and blood is considered to be a non-Newtonian fluid, and the rheological parameters (dynamic viscosity) of blood are described by a generalized power law. The Newtonian law describes behavior of magnetic particles, and the total force is represented by magnetic force and drag force. The equation of the walls of arteries and plaques, plaques and vascular walls is introduced in the form of porous media formulated by the Darcy-Forchheimer model to take into account the inertial and viscous effects on the blood stream. The motion equation describing the problem consists of three equations: Navier-Stokes equation is the fluid (blood) equation. The Darcy-Forchheimer model used for porous media phase and the Lagrangian discrete phase model (DPM) calculates the trajectory of particles [10].

Voronin et al. [11] used the multilayer composite magnetic microcapsules as targeted delivery systems under natural conditions of living organism in in vitro and in vivo studies. Visualization and capture by magnetic field of the capsules at the defined blood flow is shown in vitro in an artificial glass capillary using a wide-field fluorescence microscope. Afterwards, the capsules were visualized, successfully trapped and retained at the site of interest by the magnetic field in the bloodstream of micro-vessels of rat mesentery in vivo. In vitro experiments on a glass tube demonstrate the possibility of a locally applied nonuniform permanent magnetic field catching the capsules in the bloodstream.

In this current theoretical study, magnetic nanoparticles such as superparamagnetic iron oxide nanoparticles $\left(\mathrm{Fe}_{3} \mathrm{O}_{4}\right)$ are used. To attract the magnetic particles to targeted positions, the magnet is placed nearby one of the brunches of the branched vessel. The fluid (blood) is considered Newtonian; its flow is incompressible, laminar. The dynamics of MNPs moving in a static magnetic field in Newtonian fluid was analyzed numerically. In the model, the dominant magnetic and drag forces are used. A combination of magnetic equations for the permanent magnet field and Navier-Stokes equations for the fluid governs the equations of motion for MNPs in the flow. Using the
COMSOL Multiphysics ${ }^{\circledR}$ Simulation Program, these equations were solved numerically.

Motivation for this theoretical study to examine parameters of real complexity and real geometry magnetically operated structures, such as the distribution and treatment of magnetic drugs for unique biomedical applications [1-13] and magnetomotive laser speckle imaging [14].

\section{Formulation of the Problem}

The permanent cylindrical magnet is located outside of the branch's blood vessel. The vessel is symmetric i.e. length of branch vessel = length of original vessel.

In order to solve the problem, there are two domains in the model: first the branched blood vessel domain with a cylindrical cross-section containing the magnetic particle and the fluid (blood), and second, the permanent magnet domain. As shown in Fig. 1A, bifurcation vessel with one inlet and two outlets is the geometry used in this study. The COMSOL Multiphysics ${ }^{\circledR}$ software was used to solve flow and magnetic field equations numerically under the initial and boundary conditions, based on a finite element approach, which is a numerical technique in partial differential equations to find approximate solutions to boundary value problems (Table 1).

Table 1 Dimensions of blood vessel, magnet and particle diameter used in simulation.

\begin{tabular}{|c|c|c|}
\hline Name & Value & Description \\
\hline D_magnet & $50[\mathrm{~mm}]$ & Diameter of magnet [9] \\
\hline 1_magnet & $30[\mathrm{~mm}]$ & Length of magnet [9] \\
\hline u_max & $1[\mathrm{~mm} / \mathrm{s}]$ & $\begin{array}{l}\text { Maximum velocity of } \\
\text { fluid(blood) [11] }\end{array}$ \\
\hline$\rho$ & $\begin{array}{c}1060 \\
{\left[\mathrm{~kg} / \mathrm{m}^{3}\right]}\end{array}$ & Density of blood \\
\hline$\eta$ & $\begin{array}{l}3.5 \times 10^{-3} \\
{[\mathrm{~Pa} \times \mathrm{s}]}\end{array}$ & $\begin{array}{c}\text { Dynamic viscosity of } \\
\text { blood }\end{array}$ \\
\hline D_particle & $6[\mathrm{~nm}]$ & Diameter of particle [9] \\
\hline$\mu \mathrm{m}$ & 1.05 & $\begin{array}{l}\text { Relative permeability of } \\
\text { neodymium magnet }\end{array}$ \\
\hline
\end{tabular}

\section{Equations of Motion}

The equations describing the problem were solved numerically by using three different modules of the COMSOL Multiphysics ${ }^{\circledR}$ Software. These modules include:

1. AC/DC module to calculate the magnetic field of the permanent magnet with cylindrical cross section. A stationary magnetic field produced by a permanent magnet implanted at a specific location is described by the equations for the static magnetic field derived from the Ampere-Maxwell equation [15]. As the magnetic flux 


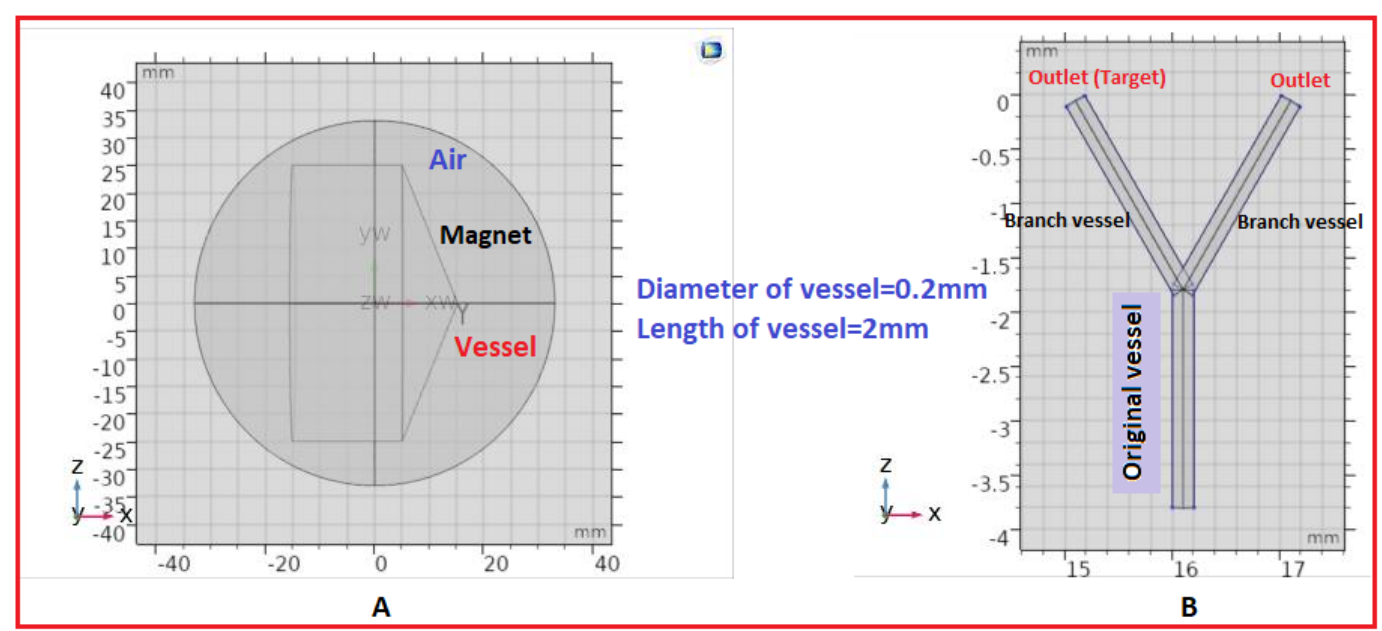

Fig. 1 Geometry domains for the model.

density $\bar{B}$ that in different domains can be described by the relation between $\bar{B} \& \bar{H}$ for the permanent magnet:

$$
\bar{B}=\mu_{0} \mu_{r} \bar{H}+\bar{B}_{r e m},
$$

where $\mu_{0}$ is the magnetic permeability in free space, $\mu_{0}=4 \pi \times 10^{-7} \mathrm{~N} / \mathrm{A}^{2}, \mu_{r}$ is the relative permeability which is a ratio of the permeability of a specific medium to the permeability of free space $\mu_{0} ; \mathrm{H}$ is the magnetic field strength; $B$ is the magnetic flux density; $\bar{B}_{\text {rem }}$ is the remanent magnetic flux density.

2. CFD module for laminar (fully developed) fluid flow such as blood in the vessel with cylindrical cross section. The motion of blood through the vessel can be expressed by incompressible Navier-Stokes equations [16], which considers the fluid as a Newtonian. At the inlet of the vessel, the fluid flow is directed in $z$-axis. No slip condition for all vessel walls as shown in Fig. 1B.

3. Particle tracing module for modeling of particle trajectories moving through the blood vessel. Magnetic particles moving in the environment inside the vessel are subjected to many different forces. These forces include magnetic force $\left(F_{M}\right)$ which is arising from magnetic field, its strong gradient created from external permanent magnet. Viscous drag force $\left(F_{D}\right)$ which is due to movement of magnetic particles with respect to the surrounding fluid, buoyance force $\left(F_{b}\right)$, gravity force $\left(F_{g}\right)$ due to the effect of gravitation on particles, inertia and particle-particle interactions. However, only major forces are considered the hydrodynamic drag and magnetophoretic force. Our model ignores inertia [17], buoyancy [18], gravitational [19], and particle-particle interaction forces because they are several orders of magnitude weaker than the magnetic force. Brownian force [20] not used in our model because the nature of this force Brownian motion describes the random motion of small particles about or under $1 \mu \mathrm{m}$ in diameter and it causes the fluctuations in particle position, also this force predicted by the viscosity of fluid and The force due to Brownian motion is dominated by a friction force. Only major forces are considered: the hydrodynamic drag [21] and magnetophoretic force [22].
The drag force for spherical nanoparticle with diameter $D$ is the Stokes drag force:

$$
\bar{F}_{D}=\frac{18 \eta}{\rho_{p} D^{2}} m_{p}\left(\bar{u}-\bar{v}_{p}\right)
$$

where $\eta$ is the viscosity of blood, $m_{p}$ is the mass of a magnetic nanoparticle, $\overline{v_{p}}$ is the vector velocity of particle, $\rho_{p}$ is the density of particle material, $D$ is the diameter for a spherical magnetic nanoparticle. The magnetophoretic force, caused by the magnetic field action on the particle, is given by:

$$
\bar{F}_{M A P}=2 \pi \mu_{r} \mu_{0} r^{3}\left[\frac{\mu_{r, p}-\mu_{r}}{\mu_{r, p}+2 \mu_{r}} \nabla H^{2}\right]
$$

$\mu_{r, p}$ is the relative permeability for magnetic particle. The magnetophoretic force in Eq. (3) is proportional to the gradient of the magnetic field power density $\nabla H^{2}$ and particle radius as $r^{3}$.

The trajectories and velocities of a magnetic nanoparticle with mass $m_{p}$ were calculated from the equation:

$$
m_{p} \frac{d \bar{v}_{p}}{d t}=\bar{F}_{t}
$$

and the total force for a particle is given as:

$$
\bar{F}_{t}=\bar{F}_{M A P}+\bar{F}_{D} .
$$

Because the mass of particle is small so the terminal velocity constant i.e. $\left(\frac{d \bar{v}_{p}}{d t}=0\right)$ so the particle velocity is given from the balancing magnetic and drag force

$$
\bar{F}_{M A P}+\bar{F}_{D}=0
$$

\section{Results and Discussions}

The cylindrical permanent magnet with magnetic field $B=0.45 \mathrm{~T}$ was applied to the bifurcation blood vessel with cylindrical cross section, the line graph explained 
the behavior of magnetic flux density along the magnet and the tip of magnet (Arc length), Figs. 2C and 2B.

It is assumed that 4500 magnetic particles with a diameter of $6 \mathrm{~nm}$ are released from the inlet into the vessel and captured by a permanent magnet with a cylindrical cross section. Drag force is the driving force, which helps to transport particles through the blood vessel. It depends on the behavior of velocity within the vessel, i.e. the velocity of particle takes the same behavior of the blood as shown in Fig. 3 .
The magnetophoretic force must overcome the drag force in the presence of magnetic field to be able to capture a particle at a desired location (target). Depending on the magnitude of the magnetophoretic force, the magnetic force could help the trajectory of particle near the magnet site, the large number of particles from the inlet is directed towards the magnet tip and thus their concentration is maximal in the space nearby to the magnet tip as shown in Figs. 4A and 4B.
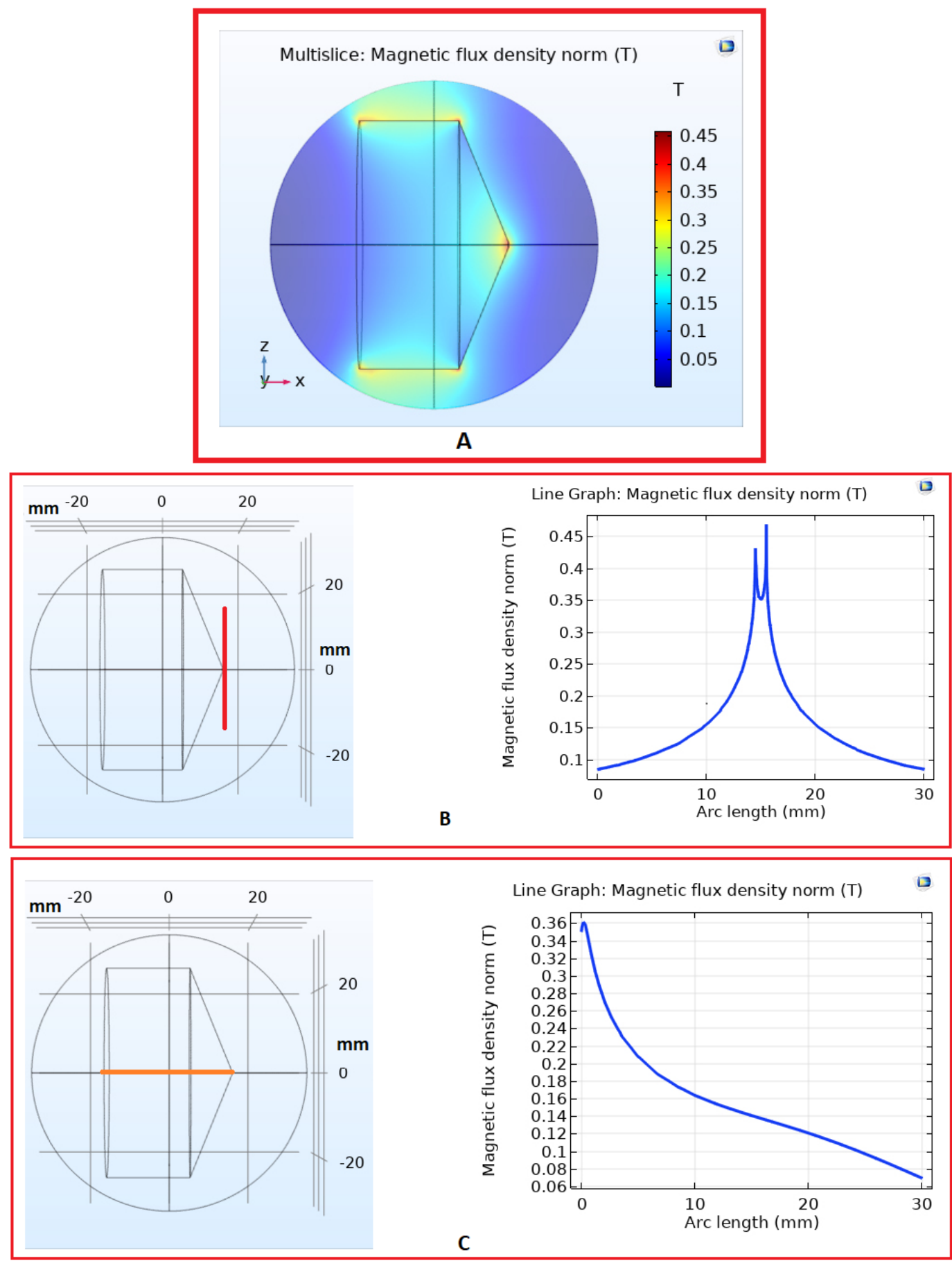

Fig. 2 Results of simulation of magnetic field of the permanent magnet with cylindrical cross section: multi-slice of magnetic flux density norm in tesla (T); line graphs (B) and (C) explained the behavior of magnetic flux density at the tip and along the length of magnet, respectively; Arc length represented by the red line as in (B) and (C). 

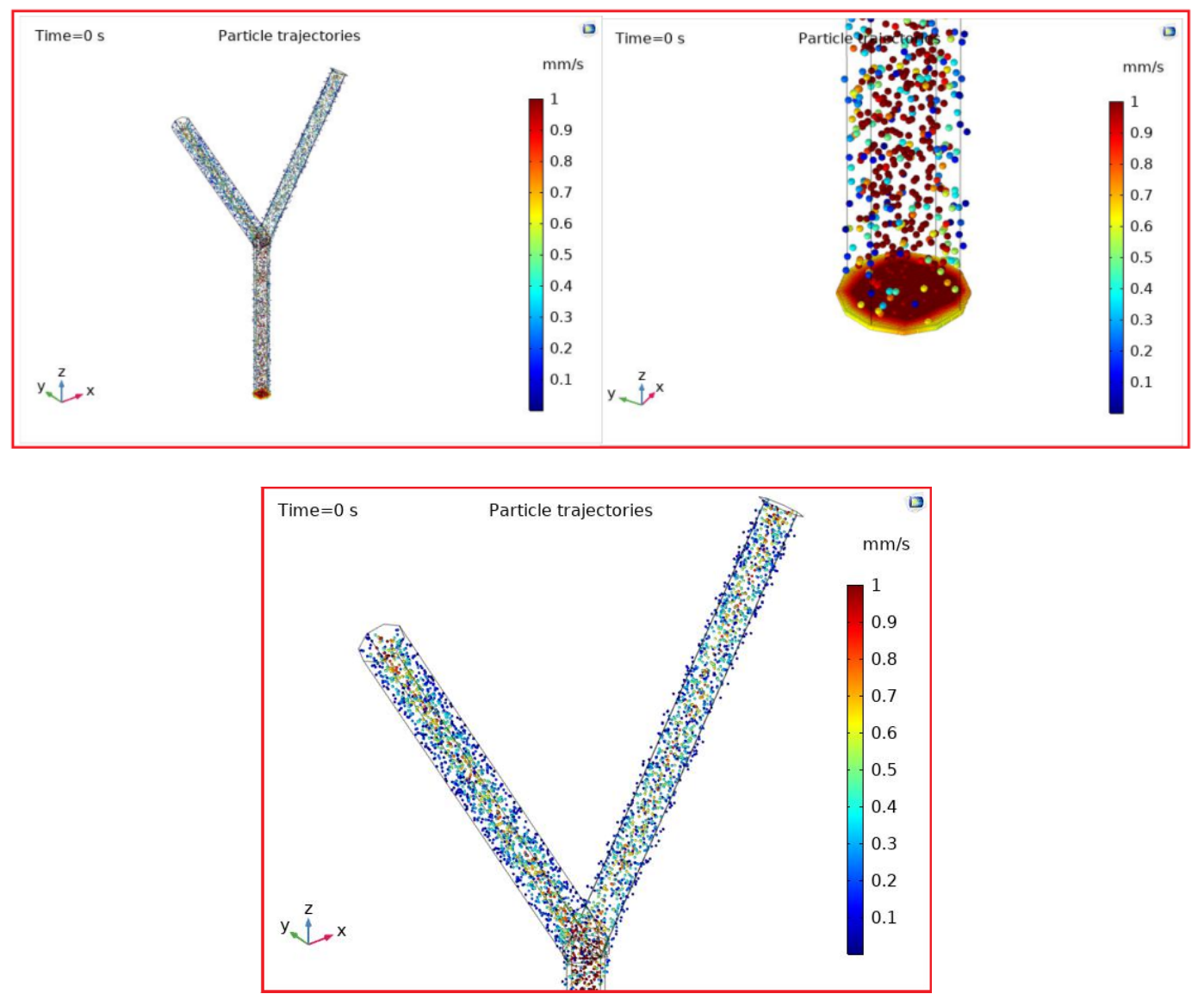

Fig. 3 Simulation result for magnetic particles in the bifurcated blood vessel. The distribution of particle velocity magnitude in the presence of drag force.

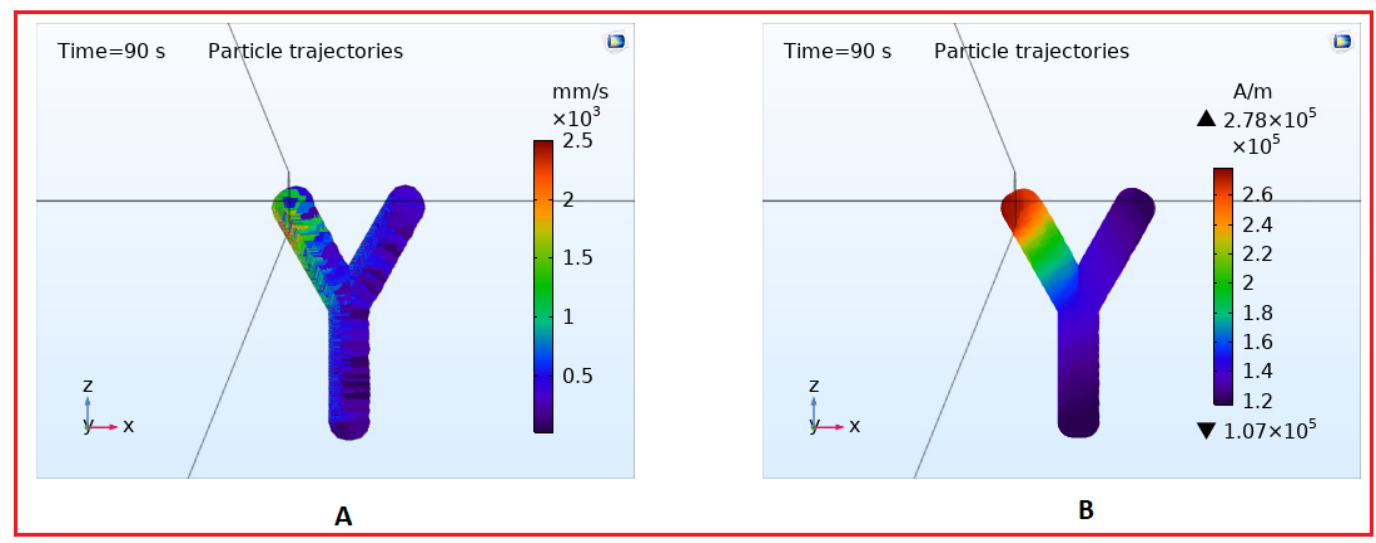

Fig. 4 Simulation result for magnetic particles in the bifurcated blood vessel. In the presence of magnetic field, the particle velocity and the concentration of particles under magnetic field effect as shown respectively in (A) and (B).

\section{Conclusion}

In this study, numerical results were obtained to clarify the action of the magnetic field created by cylindrical permanent magnet placed outside the bifurcated blood vessel on magnetic nanoparticles traveling in the blood flow of the vessel. In this model, blood is regarded Newtonian fluid, laminar flow (fully developed) and with a non-magnetized property. The dominant magnetic and drag powers are considered in the equations used for simulations. Navier Stokes equations for the blood stream and magnetic equations for a permanent magnet govern the movement equations that define the flow through the vessel. By using the COMSOL Multiphysics ${ }^{\circledR}$ modelling software, equations have been solved numerically. In many biomedical applications, including drug targeting for treatment of cancer cells and hyperthermia treatments, the results obtained in this study can be used. 


\section{Disclosures}

All authors declare that there is no conflict of interests in this paper.

\section{Acknowledgements}

This work was supported by grant of the Government of the Russian Federation 14.W03.31.0023.

\section{References}

1. S. Sukumaran, M. S. Neelakandan, N. Shaji, P. Prasad, and V. K. Yadunath, "Magnetic Nanoparticles: Synthesis and Potential Biological Applications," JSM Nanotechnology \& Nanomedicine 6(2), 1068 (2018).

2. G. Kandasamy, D. Maity, "Recent advances in superparamagnetic iron oxide nanoparticles (SPIONs) for in vitro and in vivo cancer nanotheranostics," International Journal of Pharmaceutics 496(2), 191-218 (2015).

3. K. McNamara, S. A. M. Tofail, "Nanoparticles in biomedical applications," Advances in Physics: X 2(1), 54-88 (2017).

4. M. R. Ghazanfari, M. Kashefi, S. F. Shams, and M. R. Jaafari, "Perspective of $\mathrm{Fe}_{3} \mathrm{O}_{4}$ Nanoparticles Role in Biomedical Applications," Biochemistry Research International, 7840161 (2016).

5. M. T. H.Bhuiyan, M. N. Chowdhury, and M. S. Parvin, "Potential Nanomaterials and their Applications in Modern Medicine: An Overview," ARC Journal of Cancer Science 2(2), 25-33 (2016).

6. Y.-T. Chen, A. G. Kolhatkar, O. Zenasni, S. Xu, and T. R. Lee, "Biosensing Using Magnetic Particle Detection Techniques," Sensors 17(10), 2300 (2017).

7. Y. Chen, X. Ding, Y. Zhang, A. Natalia, X. Sun, Z. Wang, and H. Shao, "Design and synthesis of magnetic nanoparticles for biomedical diagnostics," Quantitative imaging in medicine and surgery 8(9), 957-970 (2018).

8. K. McNamara, S. A. Tofail, "Nanosystems: The use of nanoalloys, metallic, bimetallic, and magnetic nanoparticles in biomedical applications," Physical Chemistry Chemical Physics 17(42), 27981-27995 (2015).

9. O. A. Sindeeva, R. A. Verkhovskii, A. S. Abdurashitov, D. V. Voronin, O. I. Gusliakova, A. A. Kozlova, O. A. Mayorova, A. V. Ermakov, E. V. Lengert, N. A. Navolokin, V. V. Tuchin, D. A. Gorin, G. B. Sukhorukov, and D. N. Bratashov, "Effect of Systemic Polyelectrolyte Microcapsule Administration on the Blood Flow Dynamics of Vital Organs," ACS Biomaterials Science \& Engineering 6(1), 389-397 (2020).

10. X. Zhang, M. Luo, P. Tan, L. Zheng, and C. Shu, "Magnetic nanoparticle drug targeting to patient-specific atherosclerosis: Effects of magnetic field intensity and configuration," Applied Mathematics and Mechanics 41(2), 349-360 (2020).

11. D. Voronin, O. Sindeeva, M. Kurochkin, O. Mayorova, I. Fedosov, O. Semyachkina-Glushkovskaya, D. Gorin, V. V. Tuchin, and G. Sukhorukov, "In vitro and in vivo visualization and trapping of fluorescent magnetic microcapsules in a blood stream," ACS Applied Materials \& Interfaces 9(8), 6885-6893 (2017).

12. E. M. Múzquiz-Ramos, V. Guerrero-Chávez, B. I. Macías-Martínez, C. M. López-Badillo, and L. A. García-Cerda, "Synthesis and characterization of maghemite nanoparticles for hyperthermia applications," Ceramics International Part A 4(1), 397-402(2015).

13. R. Di Corato, A. Aloisi, S. Rella, J.-M. Greneche, G. Pugliese, T. Pellegrino, C. Malitesta, and R. Rinaldi, "Maghemite Nanoparticles with Enhanced Magnetic Properties: One-Pot Preparation and Ultrastable Dextran Shell," ACS Applied Materials \& Interfaces 10(24), 20271-20280 (2018).

14. J. Kim, J. Oh, and B. Choi, "Magnetomotive laser speckle imaging," Journal of Biomedical Optics 15(1), 011110 (2010).

15. E. P. Furlani, Permanent Magnet and Electromechanical Device, Materials, Analysis and Applications, Academic, New York (2001).

16. R. B. Bird, R. C. Armstrong, and O. Hassager, Dynamics of Polymeric Fluids, Volume 1 Fluid Mechanics, Wiley, NY (1987).

17. L. Leal, "Particle motions in a viscous fluid," Annual Review of Fluid Mechanics 12(1), 435-476 (1980).

18. Y. Liu, W. K. Liu, T. Belytschko, N. Patankar, A. C. To, A. Kopacz, and J. H. Chung, "Immersed electro kinetic finite element method," International Journal for Numerical Methods in Engineering 71(4), 379-405 (2007).

19. J. G. Teeguarden, P. M. Hinderliter, G. Orr, B. D. Thrall, and J. G. Pounds, "Particokinetics in vitro: dosimetry considerations for in vitro nanoparticle toxicity assessments," Toxicological Sciences 95(2), 300-312 (2006).

20. S. Wang, Y. Zhou, J. Tan, J. Xu, J. Yang, and Y. Liu, "Computational modeling of magnetic nanoparticle targeting to stent surface under high gradient field," Computational mechanics 53(3), 403-412 (2004).

21. B. Kirby, Micro- and nanoscale fluid mechanics transport in microfluidic devices, Cambridge University Press, New York (2010).

22. T. B. Jones, Dielectrophoresis and magnetophoresis, Electromechanics of Particles, Cambridge University Press, New York (1995). 